\title{
Proton MR Spectroscopy of Neural Stem Cells: Does the Proton-NMR Peak at 1.28 ppm Function As a Biomarker for Cell Type or State?
}

\author{
Kai F. Loewenbrück, ${ }^{1,2}$ Beate Fuchs, ${ }^{3}$ Andreas Hermann,, ${ }^{1,2}$ Moritz Brandt, ${ }^{1,2}$ Annett Werner, ${ }^{4}$ \\ Matthias Kirsch, ${ }^{5}$ Sigrid Schwarz, ${ }^{6}$ Johannes Schwarz, ${ }^{6,7}$ Jürgen Schiller, ${ }^{3}$ and Alexander Storch ${ }^{1,2}$
}

\begin{abstract}
Recently, a peak at $1.28 \mathrm{ppm}$ in proton magnetic resonance spectroscopy ( ${ }^{1} \mathrm{H}-\mathrm{MRS}$ ) of neural stem cells (NSCs) was introduced as a noninterventional biomarker for neurogenesis in vivo. This would be an urgently needed requisite for translational studies in humans regarding the beneficial role of adult neurogenesis for the structural and functional integrity of the brain. However, many concerns have risen about the validity of the proposed signal as a specific marker for NSCs. The peak has also been related to cell-type-independent phenomena such as apoptosis or necrosis. Thus, we compared the 1.28-ppm peak in various immature stem cell populations, including embryonic stem cells, mouse embryonic fibroblasts, embryonic stem cell- and induced pluripotent stem cell-derived NSCs, ex vivo isolated embryonic NSCs, as well as mature and tumor cell types from different germ layers. To correlate the integral peak intensity with cell death, we induced both apoptosis with camptothecin and necrosis with sodium azide. A peak at $1.28 \mathrm{ppm}$ was found in most cell types, and in most, but not all, NSCH cultures, demonstrating no specificity for NSCs. The intensities of the 1.28-ppm resonance significantly correlated with the rate of apoptosis, but not with the rate of necrosis, cell cycle phase distribution, cell size, or type. Multiple regression analysis displayed a significant predictive value of the peak intensity for apoptosis only. In this context, its specificity for apoptosis as a major selection process during neurogenesis may suggest this resonance as an indirect marker for neurogenesis in vivo.
\end{abstract}

\section{Introduction}

A NIMAL RESEARCH PROVIDES EVIDENCE for both the importance of adult neurogenesis for the structural integrity and performance of the central nervous system and at the same time for the responsiveness of neural stem cells (NSCs) to internal and external (environmental) stimuli. ${ }^{1-3}$ Thus, neurogenesis is believed to be a promising therapeutic target for cell replacement strategies in both brain injury and neurodegenerative diseases. ${ }^{4-7}$ However, translational research to reproduce these findings in humans has largely been prevented by the lack of an adequate method to study neurogenesis in vivo. Whereas post mortem bromodeoxyuridine (BrdU) incorporation studies in cancer patients point to the existence of some postnatal neurogenesis in the brain, ${ }^{8,9}$ a tool for studying the physiological regulation of human neurogenesis is still unavailable. In 2007, a study by Manganas and colleagues proposed that not only NSCs possess a cell type-specific peak at $1.28 \mathrm{ppm}$ in proton magnetic resonance spectroscopy $\left({ }^{1} \mathrm{H}-\mathrm{MRS}\right)$, but also that this peak is suited to study physiological and pathological regulation of neurogenesis in both animals and humans in vivo. ${ }^{10}$ Since this publication offered the so far missing link for translational research, it received a high level of attention, but at the same time it raised several concerns on both the methodological level and the biological significance. On the one hand, it was questioned whether the singular value decomposition (SVD) spectral analysis used in this study was suitable for generating reliable data, and, on the other hand, the cell type specificity of the signal was heavily debated. ${ }^{11-14}$

\footnotetext{
${ }^{1}$ Department of Neurology, Dresden University of Technology, Dresden, Germany.

${ }^{2}$ Center for Regenerative Therapies Dresden, Dresden, Germany.

${ }^{3}$ Institute for Medical Physics and Biophysics, University of Leipzig, Leipzig, Germany.

${ }^{4}$ Department of Neuroradiology, Institute of Radiology, Dresden University of Technology, Dresden, Germany.

${ }^{5}$ Department of Neurosurgery, Dresden University of Technology, Dresden, Germany.

${ }^{6}$ Department of Neurology, University of Leipzig, Leipzig, Germany.

${ }^{7}$ Division of Biophysics, California Institute of Technology, Pasadena, California.
} 
The proposed NSC-specific proton magnetic resonance spectroscopy ( $\left.{ }^{1} \mathrm{H}-\mathrm{MRS}\right)$ peak has long been known as a signal derived from methylene groups in fatty acyl residues in mobile lipids, most likely located in intracellular lipid bodies. ${ }^{15-17}$ More importantly, there is substantial information available on the correlation of these intracellular lipid bodies and their associated ${ }^{1} \mathrm{H}-\mathrm{MRS}$ signal to different biological phenomena, particularly apoptosis. ${ }^{16,18}$ It is unclear from the above-mentioned study by Manganas and coworkers how the proposed NSC-specific signal at $1.28 \mathrm{ppm}$ is related to the already known biological significance of methylene group-derived lipid resonances and whether it can be distinguished from these signals at all. Indeed, a recently published study rather put the cell specificity of this signal and its potential distinction from methylene groupderived resonances in doubt. ${ }^{14}$

Together, it remains enigmatic whether there is a specificity of the signal at $1.28 \mathrm{ppm}$ in ${ }^{1} \mathrm{H}$-MRS for NSCs or whether this signal or adjacent/overlapping signals are related to various forms of cell degradation or death independent of the cell type. Thus, we studied the relevance of this peak using high-field ${ }^{1} \mathrm{H}-\mathrm{MRS}$ in a wide set of undifferentiated and maturely differentiated cell populations of all germinal sheets and quantitatively correlated it with the rate of apoptosis or necrosis as different forms of cell death.

\section{Materials and Methods}

\section{Cell culture}

Cells were cultured according to established protocols for the respective cell type. Fetal cortical NSCs were isolated from embryonic C57/BL6 mouse brain at embryonic day 14 (E14) and cultured in neurospheres as described in Milosevic et al. ${ }^{19}$ These neurosphere cultures were highly enriched NSC cultures with $91.4 \pm 9.2 \%$ of nestin ${ }^{+}$NSCs $(n=5)$. The protocol used for mixed primary neural cultures containing both glial and neuronal cells was reported in Freudenberg et al. $^{20}$ Pure astrocytic cultures were prepared according to Song et al., ${ }^{21}$ embryonic stem (ES) cells and ES-cell derived NSCs according to Kawasaki et al., ${ }^{22}$ and induced pluripotent stem (iPS) cell-derived NSCs and embryonic fibroblasts as described in Kim et al. ${ }^{23}$ MN9D cells were grown according to Heller et al. ${ }^{24}$ and melanoma and glioma cell lines according to Kirsch et al. ${ }^{25}$ and Uo et al., ${ }^{26}$ respectively. Note that all cells are of murine origin.

\section{Camptothecin and sodium azide treatment}

For induction of apoptosis, camptothecin (Sigma-Aldrich, Hamburg, Germany) was added to the respective cultures at $5 \mu \mathrm{M}$ final concentration $18 \mathrm{hr}$ before spectroscopic analysis, as described in Uo et al. ${ }^{27}$ Necrosis was induced by sodium azide (Sigma-Aldrich) $3 \mathrm{hr}$ before analysis at final concentrations of 46,115 , and $230 \mathrm{mM}$ according to the specifications described in Lizard et al. ${ }^{28}$

\section{Cell preparation for spectroscopic analysis}

Cells were converted into a single-cell suspension by treatment with $0.05 \%$ trypsin-EDTA (Invitrogen, Karlsruhe, Germany) for $10 \mathrm{~min}$ at $37^{\circ} \mathrm{C}$, followed by a 10 -min treatment with a mixture of $40 \mathrm{mg}$ of DNase, $150 \mathrm{mg}$ of trypsin inhibitor, and $1.81 \mathrm{~g}$ of $\mathrm{MgSO}_{4}$ (all from Sigma-Aldrich) and subsequent trituration with fire-polished glass pipettes. Cells were then washed twice with phosphate-buffered saline (PBS) and adjusted to a concentration of $10 \times 10^{6}$ cells $/ \mathrm{mL}$. To $500 \mu \mathrm{L}$ of the cell preparation, $50 \mu \mathrm{L}$ of trimethylsilylpropionate $(T M P)$ in pure deuterium oxide $\left(\mathrm{D}_{2} \mathrm{O}\right)$ was added as a frequency lock and internal standard (both from SigmaAldrich). Cell suspensions were transferred to nuclear magnetic resonance (NMR) sample tubes (VWR International, Darmstadt, Germany) and kept on ice until ${ }^{1} \mathrm{H}-\mathrm{MRS}$ analysis.

\section{NMR acquisition}

One-dimensional ${ }^{1} \mathrm{H}-\mathrm{NMR}$ spectra of the aqueous suspensions of cells $(0.55 \mathrm{~mL}, \mathrm{pH} 7.25)$ containing $10 \% \mathrm{D}_{2} \mathrm{O}$ as a field frequency lock were measured using a 600-MHz Bruker Avance NMR spectrometer (Bruker Biospin, Rheinstetten, Germany). Spectral analysis was conducted using XWinNMR, v. 3.5. In all experiments, the temperature was maintained at $35^{\circ} \mathrm{C}$ and $\mathrm{pH}$ at 7.25 . The spectra were acquired as free induction decay (FID, 32,768 points in a spectral width of $8,389.3 \mathrm{~Hz}$, readout time of $1.95 \mathrm{sec}$, repetition time of $2 \mathrm{sec}$ and 128 averages). To minimize the large water peak, the water signal was presaturated with a low-power radiofrequency pulse. $^{9}$ Exposure of cell cultures to these MRS conditions did not influence their apoptosis rates (data not shown).

\section{Data processing/spectral analysis}

Processing of measured data was performed using the TopSpin software (BRUKER BioSpin, v. 1.3). Before Fourier transformation, FIDs were processed with a line-broadening function of $1.0 \mathrm{~Hz}$. Then ${ }^{1} \mathrm{H}-\mathrm{NMR}$ spectra were phase and baseline corrected for distortions and referenced to trimethylsilylproprionate (TSP; chemical shift $0.00 \mathrm{ppm})$. No significant broadening of the TSP resonance was detected in the presence of the investigated cells. After peak picking and integration, a deconvolution (mixed line shapes of Lorentzian and Gauss) was performed, followed by the normalization of the individual integral values using the TSP peak (defined constant concentration).

\section{Measuring of apoptosis, cell cycle distribution, and necrosis}

Immediately after ${ }^{1} \mathrm{H}$-MRS analysis, the cell samples were tested for the respective level of apoptosis and necrosis. Determination of apoptotic cell fraction and cell cycle distribution was carried as described in Nicoletti et al. ${ }^{29}$ and Glick et al. ${ }^{30}$ Briefly, $1 \times 10^{5}$ of the total cells measured were washed once with PBS and then resuspended in $300 \mu \mathrm{L}$ hypotonic lysis buffer $(0.1 \%$ sodium citrate, $0.1 \%$ Triton $\mathrm{X}-100$, $50 \mu \mathrm{g} / \mathrm{mL}$ propidium iodide [Invitrogen, Karlsruhe, Germany]). After an incubation period of $30 \mathrm{~min}$ on ice and in the dark, cells were directly analyzed using fluorescenceactivated cell sorting (FACS; LSRII FACS analyzer, Becton \& Dickinson, Heidelberg, Germany). Testing for necrosis was performed using the LIVE/DEAD ${ }^{\circledR}$ Fixable Dead Cell Stain Kit according to the manufacturer's specifications (L34955, Invitrogen, Karlsruhe, Germany).

\section{Statistical analysis}

Statistics were calculated using SPSS software Version 16.0 (SPSS, Chicago, IL). Significant differences between two or 
more groups were determined using the Student two-sided unpaired $t$-test or analysis of variance (ANOVA), respectively. A $p$ value $<0.05$ was accepted as significant. The Pearson correlation coefficient or multiple linear regression modeling using different exclusion criteria was used to test correlation and predictive value for the peak at $1.28 \mathrm{ppm}$ as the dependent variable. Individual values were used for statistical analysis, with the exception of cell diameter. Cell diameter showed little variance between different samples of the same cell type. Therefore, the mean cell diameter was used in those samples where individual values had not been collected.

\section{Results}

\subsection{8-ppm peak expression in high-field ${ }^{1} \mathrm{H}-\mathrm{MRS}$ spectra of living cells during neural developmental specification}

Figure 1 shows a high-field ${ }^{1} \mathrm{H}$-MRS spectrum of a living murine fetal (E14) neurosphere culture of highly purified NSCs as a representative spectrum from the various stem cell populations investigated in this study. The peak of TSP at $0.00 \mathrm{ppm}$ was used as a frequency lock and internal standard for quantitative comparisons between the different samples. Peaks of metabolites characteristic for quickly dividing cell populations, such as choline, were detected in addition to different amino acids (succinate, glutamate, alanine, valine) and lactate as the end product of anaerobic glycolysis. Other studies investigating the chemical footprint of different cell types typically do not work with intact cells, but rather with cell constituents after cell degradation and lyophilization. ${ }^{31}$ This approach allows for a much more detailed study of the chemical constituents than is possible by the directly ex vivo spectra recorded in this study. However, it also prevents the analysis of signal changes related to biological activity or the physicochemical environment of different metabolites in intact cells.

We studied 1.28-ppm peak intensities over all major steps of neural development in vitro from pluripotent embryonic stem (ES) cells over NSCs of various origins (ES cell- and induced pluripotential stem [iPS] cell-derived NSCs, primary fetal cortical NSCs) to mature neural cell cultures, including maturely differentiated NSCs, primary mixed cortical cultures (consisting of glial and neuronal cell types), and primary astrocytes. The peak of interest at $1.28 \mathrm{ppm}$ could readily be detected in the spectra of all mentioned cell populations without any systematic change of integral peak intensity with respect to the developmental stage of the cell population (Figs. 2A,B). Moreover, glioma cells and the MN9D cell line as well as nonneural cell types, such as embryonic fibroblasts or melanoma cells, also expressed the 1.28-ppm peak clearly above the background noise level (Figs. 2A,B). Because the peak at $1.28 \mathrm{ppm}$ as a lipid-derived signal could be related to differences in the net lipid content of cells of different sizes, we compared peak intensities and cell diameters, not finding any correlation between these two parameters (Fig. 2C; $r=0.14, p=0.54$, Pearson correlation coefficient). Besides this lack of correlation with cell size, qualitative and quantitative comparisons of 1.28-ppm peak intensities with cell type neither revealed any specificity of the peak for undifferentiated stem cells nor for neural stem cells and their progeny (Fig. 2).

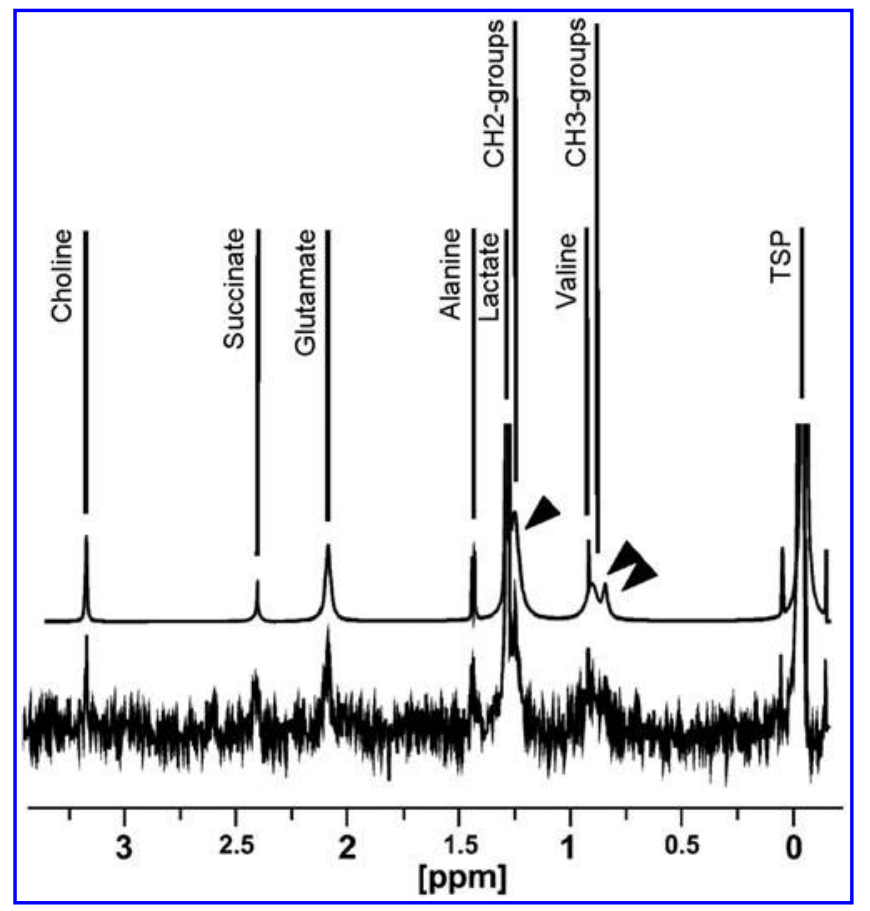

FIG. 1. Representative high-field proton magnetic resonance spectroscopy $\left({ }^{1} \mathrm{H}-\mathrm{MRS}\right)$ spectrum of a neurosphere culture of embryonic neural stem cells (NSCs) derived from embryonic cortical tissue dated embryonic day 14 (E14). The lower trace represents the original spectrum whereas the upper trace shows its computed fit, which was used for quantitative analysis. Besides typical metabolites, such as choline, succinate, glutamate, alanine, lactate, and valine, lipid-derived signals are detected both around $1.28 \mathrm{ppm}$ (arrowhead) and between 0.88 and $0.94 \mathrm{ppm}$ (two arrowheads), displaying the typical broader shape of lipid-derived peaks. Whereas the peak at $1.28 \mathrm{ppm}$ has been described to originate from mobile methylene $(\mathrm{CH} 2)$ groups in membrane-associated lipids organized in so called internal lipid bodies, the peaks between 0.88 and 0.94 ppm have been related to methyl $(\mathrm{CH} 3)$ groups in membrane-associated lipids. The intensity of both signals is known to change in a variety of biological processes, such as apoptosis, cell degeneration in general, or inflammation. The peak at $0.00 \mathrm{ppm}$ is generated by trimethylsilylpropionate (TSP) and used as an internal standard.

\section{Effects of apoptosis and cell cycle distribution on peak intensity at $1.28 \mathrm{ppm}$ in various cell systems}

In contrast to the unspecific nature of the 1.28-ppm peak with respect to the cell type and its developmental stage, comparison of the integral signal intensity with apoptosis showed a clear correlation of peak intensity with spontaneous apoptosis rate. In untreated cell cultures, cell types with a low integral intensity of the peak at $1.28 \mathrm{ppm}$, such as astrocytic or primary cortical cultures, were without exception those that had low levels of spontaneous apoptosis (Fig. $2 \mathrm{~A}, \mathrm{~B})$. Thus, we quantitatively measured the correlation between peak intensities at $1.28 \mathrm{ppm}$ and apoptotic cell death in various cell types. Apoptosis was induced by an 18-hr treatment with camptothecin, a frequently used potent inductor of apoptosis in a variety of cell types. ${ }^{32}$ The level of apoptosis was measured by the intensity of the sub- $G_{1}$ peak 


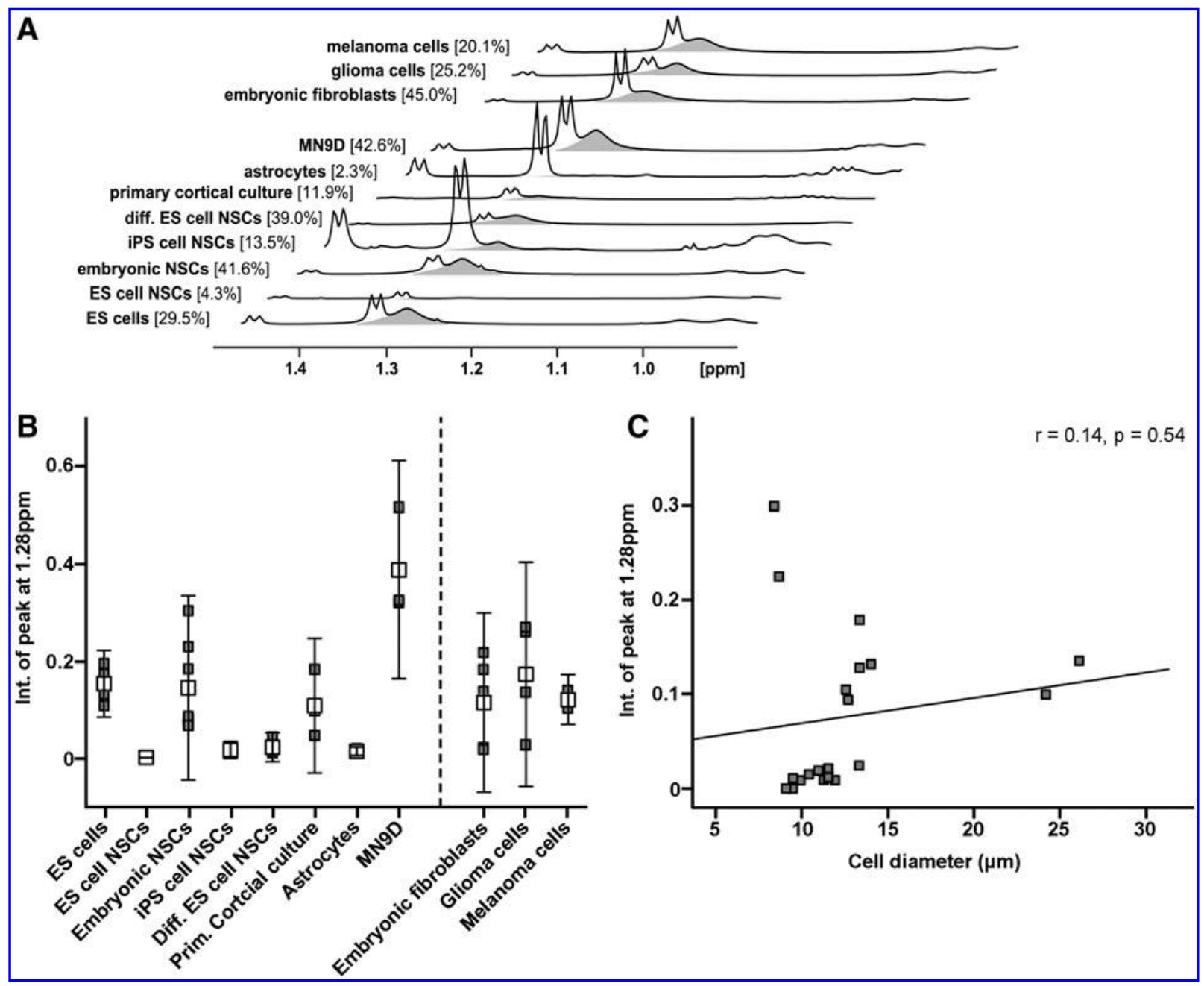

FIG. 2. Intensity of the resonance at $1.28 \mathrm{ppm}$ in proton magnetic resonance spectroscopy $\left({ }^{1} \mathrm{H}-\mathrm{MRS}\right)$ in living cell cultures is not correlated with cell type or neuro-developmental stage. (A) Mean spectra of all untreated samples of a cell type are displayed ordered according to the respective developmental stage, going from undifferentiated embryonic stem (ES) cells over neural stem cells (NSCs) of different origin (derived from ES cells, fetal cortical brain cultures, or from induced pluripotent stem [iPS] cells) to differentiated neural cell types (primary cortical cultures, astrocytes, or MN9D dopaminergic cell line). In the upper part of $\mathbf{A}$, the mean spectra of two different tumor cells (glioma and melanoma), as well as of embryonic fibroblasts as an example of a nonneural cell type, are displayed. The area are under the curve of the peaks at $1.28 \mathrm{ppm}$ is marked in grey; the percentage of apoptotic cells is shown as percentage in squared brackets. No correlation between the intensity of the resonance at $1.28 \mathrm{ppm}$ and cell type or neuro-developmental cell stage becomes apparent. Only some NSC cultures clearly express the peak, in addition to an expression of the peak by both differentiated neuronal cultures (MN9D) and cells of nonneural origin (embryonic fibroblasts). (B) Intensities of the resonance at $1.28 \mathrm{ppm}$ of all cell types ordered according to their neuro-developmental stage, going from pluripotent embryonic stem cells to primary cortical cultures consisting mostly of differentiated mature brain cells. Separated by a dashed line, the resonances of tumor cells and nonneuronal cells are displayed on the right. Values for the individual cell samples tested are shown as small grey squares, whereas mean values for a cell type are displayed as larger white squares (including the standard deviation). As in A, no change of intensity of the 1.28-ppm resonance in relation to the developmental stage level of the cell type tested becomes apparent. (C) Correlation of resonance intensities at $1.28 \mathrm{ppm}$ with cell diameters of all cell samples is displayed. No significant correlation could be detected between these parameters (parameters shown are from the Pearson correlation).

in propidium iodide (PI)-FACS analysis of degraded hypodiploid nuclei as a common final path of different forms of apoptosis (Fig. 3A). ${ }^{29}$ Treatment with camptothecin resulted in a highly significant increase of the apoptotic culture rate only in some cultures (Figs. 3 and 4A-C), whereas other cell types were resistant to the treatment and showed no significant increase in apoptosis (Figs. 3 and 4D-F). Consistent with the observation in untreated cells, significant increase of 1.28-ppm peak intensities was exclusively observed in cell cultures with significant induction of apoptosis (Fig. 4).

We then calculated statistical correlations between all cell types (treated with camptothecin or untreated) and the measured apoptotic rate. Multiple linear regression analysis using different exclusion criteria revealed that the peak intensity at $1.28 \mathrm{ppm}$ was significantly determined by the level of apoptosis only (Fig. 5A; $r^{2}=0.6, \beta=0.78, p<0.001$ ), and 


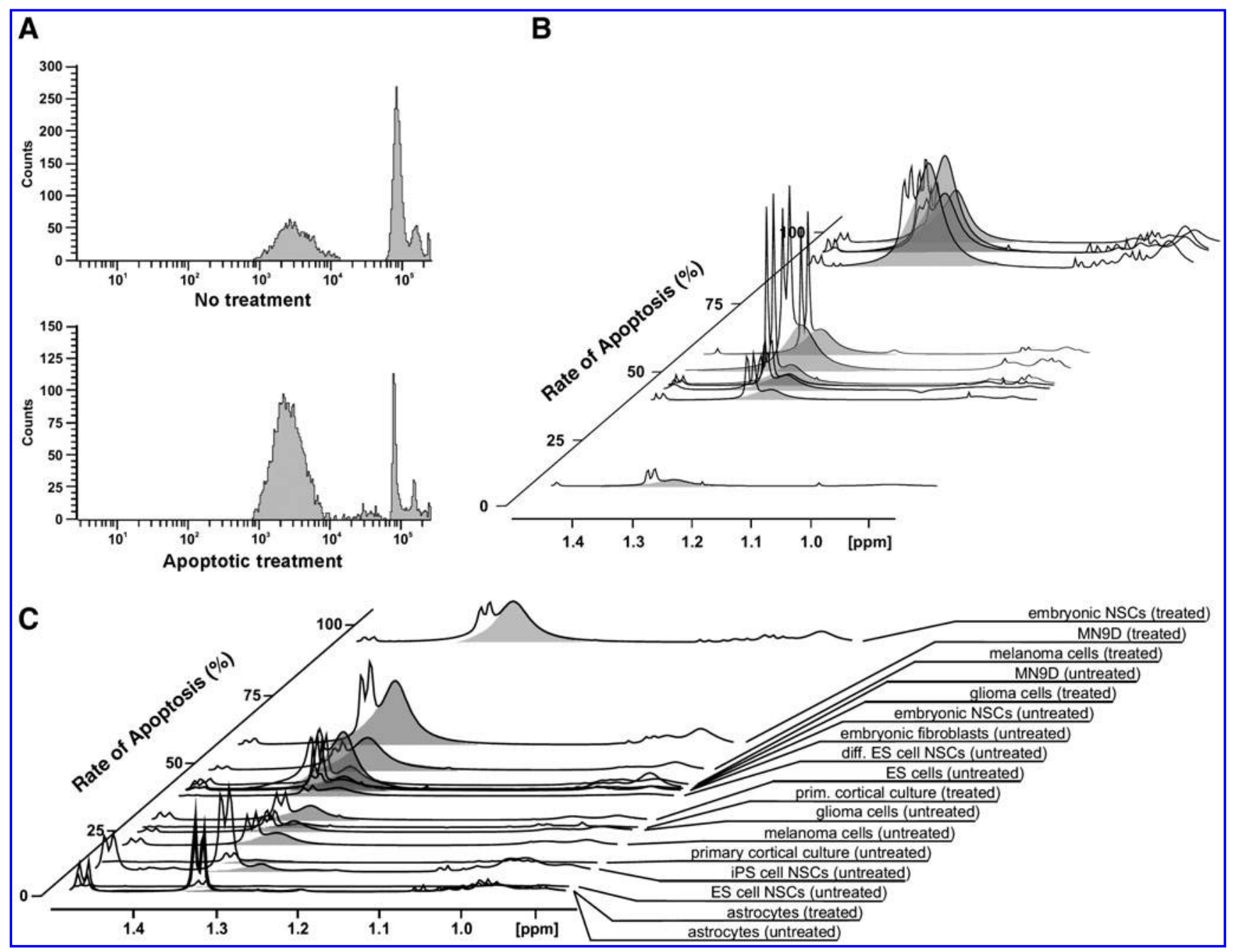

FIG. 3. Effects of apoptosis on 1.28-ppm peak intensity in proton magnetic resonance spectroscopy ( $\left.{ }^{1} \mathrm{H}-\mathrm{MRS}\right)$. (A) Expression of the sub- $\mathrm{G}_{1}$ peak as a measure of apoptosis either spontaneously or after apoptotic treatment with camptothecin in two representative cultures of embryonic neural stem cells (NSCs). (B) ${ }^{1} \mathrm{H}-\mathrm{MRS}$ spectra of different cultures of embryonic NSCs are displayed as a function of their apoptotic rate. The differences in apoptosis either were spontaneous, or induced by an 18-hr treatment with camptothecin ( $5 \mu \mathrm{M}$ final concentration). A constant increase in the integral of the peak at 1.28 ppm was detected with increasing fractions of apoptotic cells in the cultures. (C) Mean spectra of various cell types were generated for the untreated samples, as well as for the samples treated with the apoptosis-inducing agent camptothecin and ordered according to their apoptotic rate. Again, samples that had a low spontaneous rate of apoptosis or were resistant to the treatment with camptothecin (astrocytes) displayed integral intensities at $1.28 \mathrm{ppm}$ at the detection limit, whereas cell types with a high spontaneous or chemically induced apopototic rate also had high intensities of the integral of the peak at 1.28 ppm. ES cell, Embryonic stem cell; iPS cell, induced pluripotential cell.

not by any cell type (Fig. 5B; $p>0.05$ for all), cell diameter (Fig. 5C; $p=0.45$ ) or treatment with camptothecin (not shown; $p=0.23$ ). Previous reports suggested that the ratio between methylene-derived signals around $1.28 \mathrm{ppm}$ and methyl groups-derived signals around $0.9 \mathrm{ppm}$ provides a reliable marker for apoptosis. ${ }^{18}$ The results of linear regression modeling of the methylene/methyl $(\mathrm{CH} 2 / \mathrm{CH} 3)$ ratio resulted in similar results for the predictive value of apoptosis as in the respective analysis comparing 1.28 -ppm peak integral intensities only with the level of apoptosis (Fig. 5D). However, using the $\mathrm{CH} 2 / \mathrm{CH} 3$ ratio, an accompanying predictive value for the $\mathrm{CH} 2 / \mathrm{CH} 3$ ratio could be found in addition to apoptosis for ES cell type, but not for all other cell types, cell diameter, or treatment with camptothecin (results of multiple regression analysis: $r^{2}=0.75$; apoptosis, $\beta=0.70, p<0.001$; ES cells, $\beta=0.44, p=0.03$; all other variables $p>0.05$ ).

Despite the close interaction between apoptosis and the cell cycle and the observation of a significant negative correlation between the proportion of cells in apoptosis and in $\mathrm{G}_{0} / \mathrm{G}_{1}$ phase in this study $(r=-0.446, p=0.001$, Pearson correlation), no correlation could be found between the peak intensity at $1.28 \mathrm{ppm}$ and different phases of the cell cycle as distinguished by FACS-based cell cycle analysis (Fig. 5G-I; $p$ values for all cell cycle phases $>0.05$, Pearson correlation). However, the $\mathrm{CH} 2 / \mathrm{CH} 3$ ratio as an alternative MRS marker for apoptosis showed a slight negative correlation with the proportion of cells in $\mathrm{G}_{0} / \mathrm{G}_{1}$ phase $(r=-0.401, p=0.04$, Pearson correlation). 

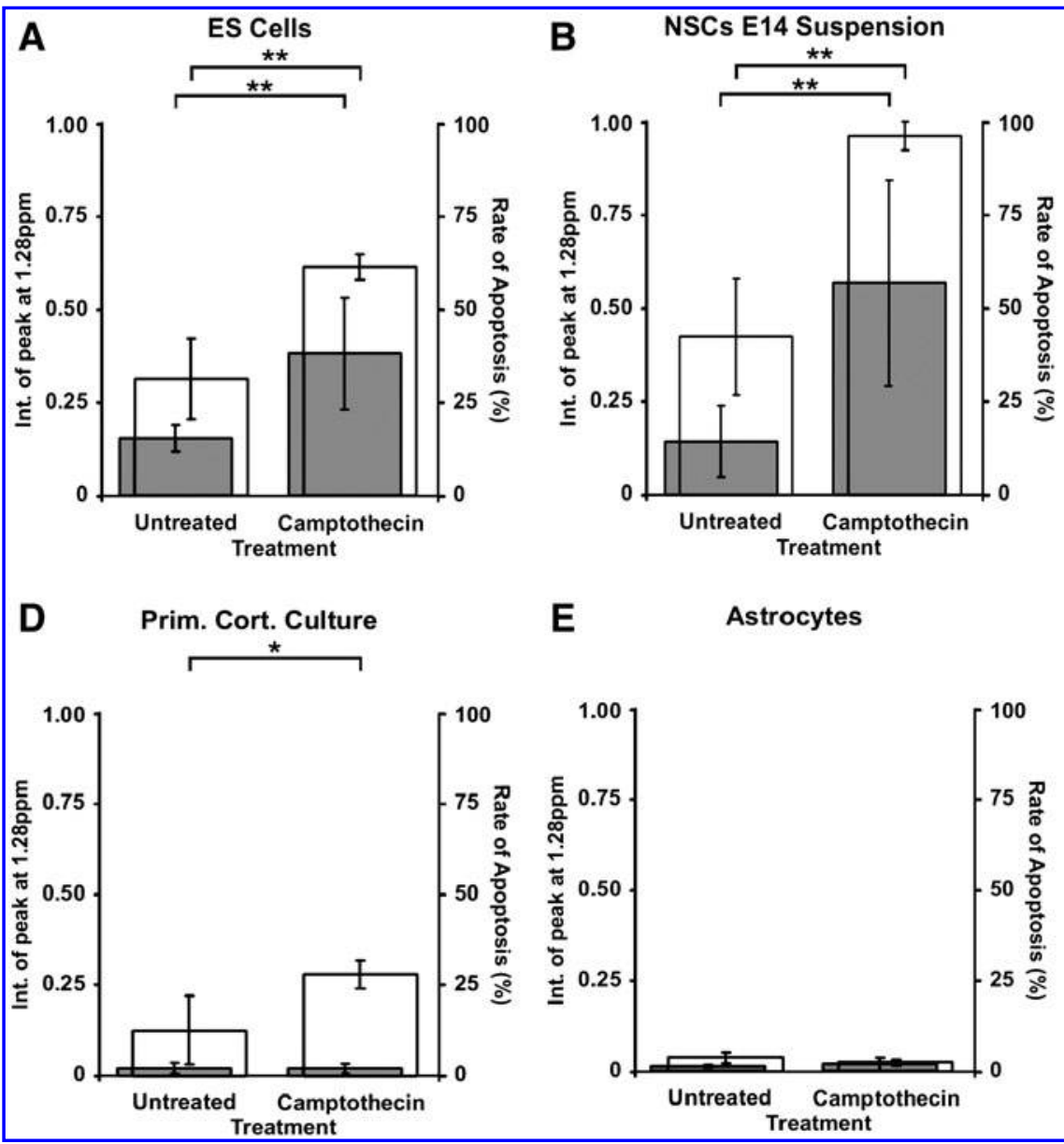

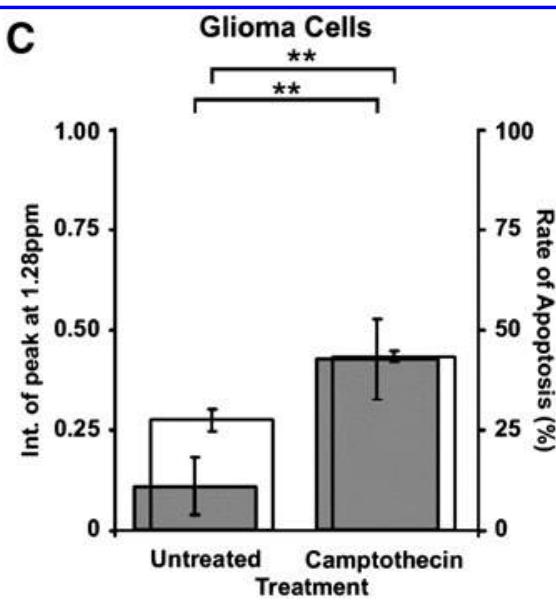

$\mathbf{F}$
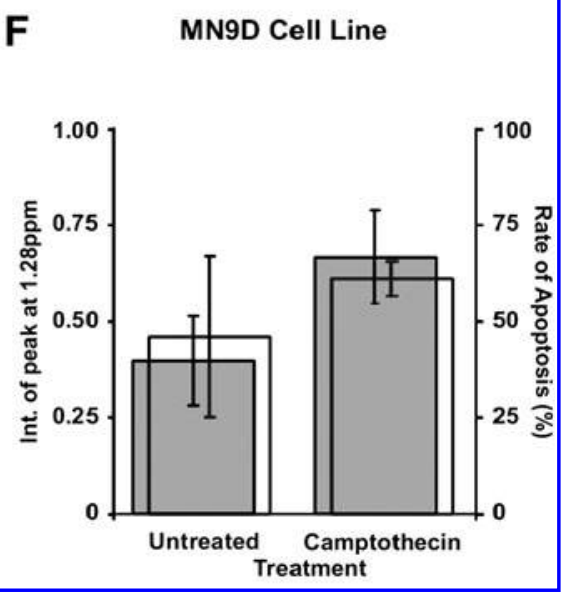

FIG. 4. Quantitative comparison of intensities of the integral of the peak at 1.28 ppm and apoptotic rates. In the upper part of the figure $(\mathrm{A}-\mathrm{C})$, cell cultures are displayed where the treatment with camptothecin lead to a significant increase in apoptosis, accompanied by a corresponding significant increase in integral intensities at 1.28 ppm. (Grey bars) Mean integral intensities at $1.28 \mathrm{ppm}$ and refer to the $y$ axis on the left side; (white bars) mean level of apoptosis of the treated and untreated cultures and refer to the $y$ axis on the right side. Whereas the treatment with camptothecin of embryonic stem (ES) cells (A), embryonic neural stem cells (NSCs) (B), and glioma cells (C) resulted in a significant induction of apoptosis ( $p<0.01$ for all) and was accompanied by a significant induction of integral intensities at $1.28 \mathrm{ppm}$, the treatment of primary cortical cultures (D), astrocytes (E), and MN9D as a dopaminergic neural cell line (F), either had no effect on the apopotic rate, or to a significance level of $p<0.05$ only, accompanied by no increase in integral intensities at 1.28 ppm. (*) Significant different results with $\left.p<0.05 ;{ }^{* *}\right)$ significant results with $p<0.01$.

Prediction by the independent variables in this study in a linear regression model was possible for the peak at $1.28 \mathrm{ppm}$ only and not for two other metabolites detected by ${ }^{1} \mathrm{H}-\mathrm{MRS}$, namely lactate and choline, showing no significant prediction of these metabolites by apoptosis rate, cell type, diameter or treatment (Figs. 5E,F; multiple regression analysis: $r^{2}=0.20, p=0.06$ and $-0.09, p=0.78$ ).

\section{Effects of necrosis on peak intensity at $1.28 \mathrm{ppm}$ in fetal cortical NSCS}

Methylene group-derived signals in the spectral region at $1.28 \mathrm{ppm}$ had also been suggested to be related to necrosis as an uncontrolled form of cell death. ${ }^{16}$ Therefore, we compared the intensities of the peak at $1.28 \mathrm{ppm}$ in fetal cortical NSC cultures with the level of necrosis (Fig. 6). Necrosis was induced with sodium azide, which deprives cells of their energy supply by blocking cytochrome $c$ oxidase. ${ }^{29}$ Although this treatment resulted in a nearly complete induction of necrosis $(93.5 \pm 2.2 \%$ in the samples treated with the highest concentration of $230 \mathrm{mM}(n=4$; Fig. $6 \mathrm{~A}, \mathrm{C})$, no increase in the 1.28 peak intensity was detected (Fig. 6B,C). Accordingly, there was no correlation between the peak intensity at $1.28 \mathrm{ppm}$ and the level of necrosis in this cell type (Fig. 6D; $r=0.05, p=0.86$, Pearson correlation coefficient).

\section{Discussion}

We aimed at understanding the biological relevance of the ${ }^{1} \mathrm{H}-\mathrm{MRS}$ signal at $1.28 \mathrm{ppm}$ in living cells, which was proposed as a NSC-specific signal and how this signal relates to the known biological significance of lipid-derived signals located precisely in this spectroscopic region. ${ }^{10}$ Our results did not reveal any correlation of the peak at $1.28 \mathrm{ppm}$ with NSCs or any other cell type tested, but showed a strong correlation of the peak with apoptotic cell death. We also tested the peak intensity at different developmental stages from pluripotent ES or iPS cells over NSCs to differentiated 


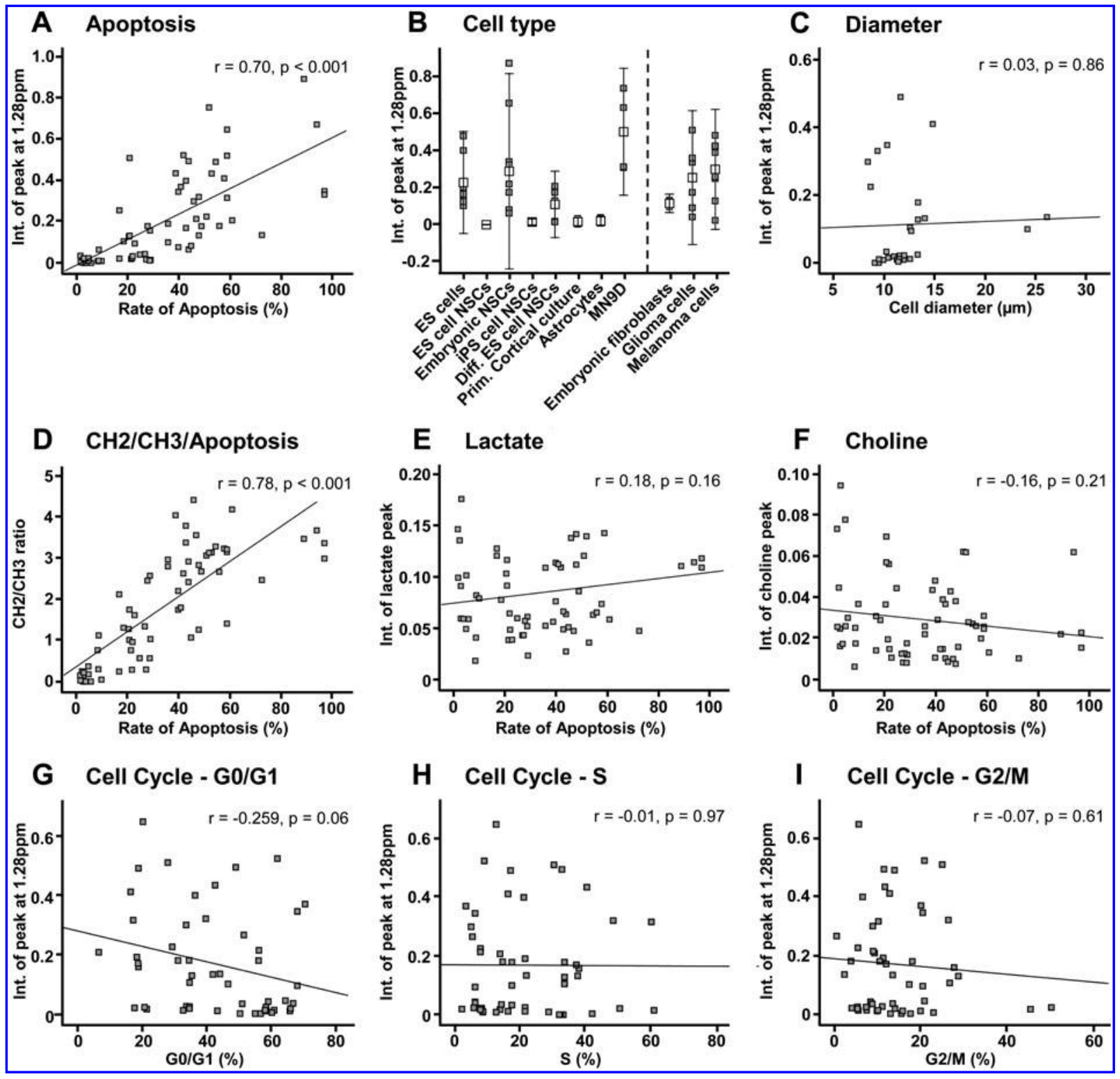

FIG. 5. Multiple linear regression analysis of integral intensities at $1.28 \mathrm{ppm}$ and various cell parameters. In multiple regression analysis, a highly significant predictive value for integral intensities at 1.28 ppm was found for level of apoptosis (A). In contrast, no significant predictive values could be detected for cell type (B), cell diameter (C), or treatment with camptothecin (not shown). (D) A positive predictive value for the ratio of integral peak intensities of methylene (CH2) groups located at $1.28 \mathrm{ppm}$ and methyl $(\mathrm{CH} 3)$ groups located between 0.8 and $0.94 \mathrm{ppm}$ was also detected. No significant predictive value could be found in the same linear multiple regression model for two other metabolites typically detected by proton magnetic resonance spectroscopy $\left({ }^{1} \mathrm{H}-\mathrm{MRS}\right)$, lactate $(\mathbf{E})$, or choline (F). (G-I) In addition, no significant correlation existed between integral peak intensities at $1.28 \mathrm{ppm}$ and different cell cycle phases as measured by fluorescence-activated cell sorting [FACS]). The Pearson correlation coefficients for the respective variables are displayed In the upper right part of each graph.

neuronal cells types, but could not find any specificity for any of these developmental stages. Multiple regression analysis displayed a significant predictive value of the peak intensity for apoptosis only, but not for necrosis, cell size, or cell type. Our data do not support the 1.28-ppm peak in ${ }^{1} \mathrm{H}$ MRS as a direct biomarker for NSCs, but its specificity for apoptosis as a major selection process during neurogenesis proposes it as an indirect marker of neurogenesis in vivo.

The results of our study are in line with one previous study on the impact of apoptosis on the peak intensity at
1.28 ppm in NSCs. ${ }^{14}$ The authors showed that apoptosis induction by menadione increased the peak intensity in all tested cell types and that it was not present in the absence of apoptosis, but they did not measure the actual apoptosis rate in their cultures and thus were not able to correlate the 1.28ppm peak intensity with the rate of apoptosis. However, quantification of the apoptosis rate is of great importance because there are large differences in both the spontaneous apoptosis rate and the responsiveness to toxic apoptosis induction between various cell lines (Fig. 4). ${ }^{32}$ The mechanisms 


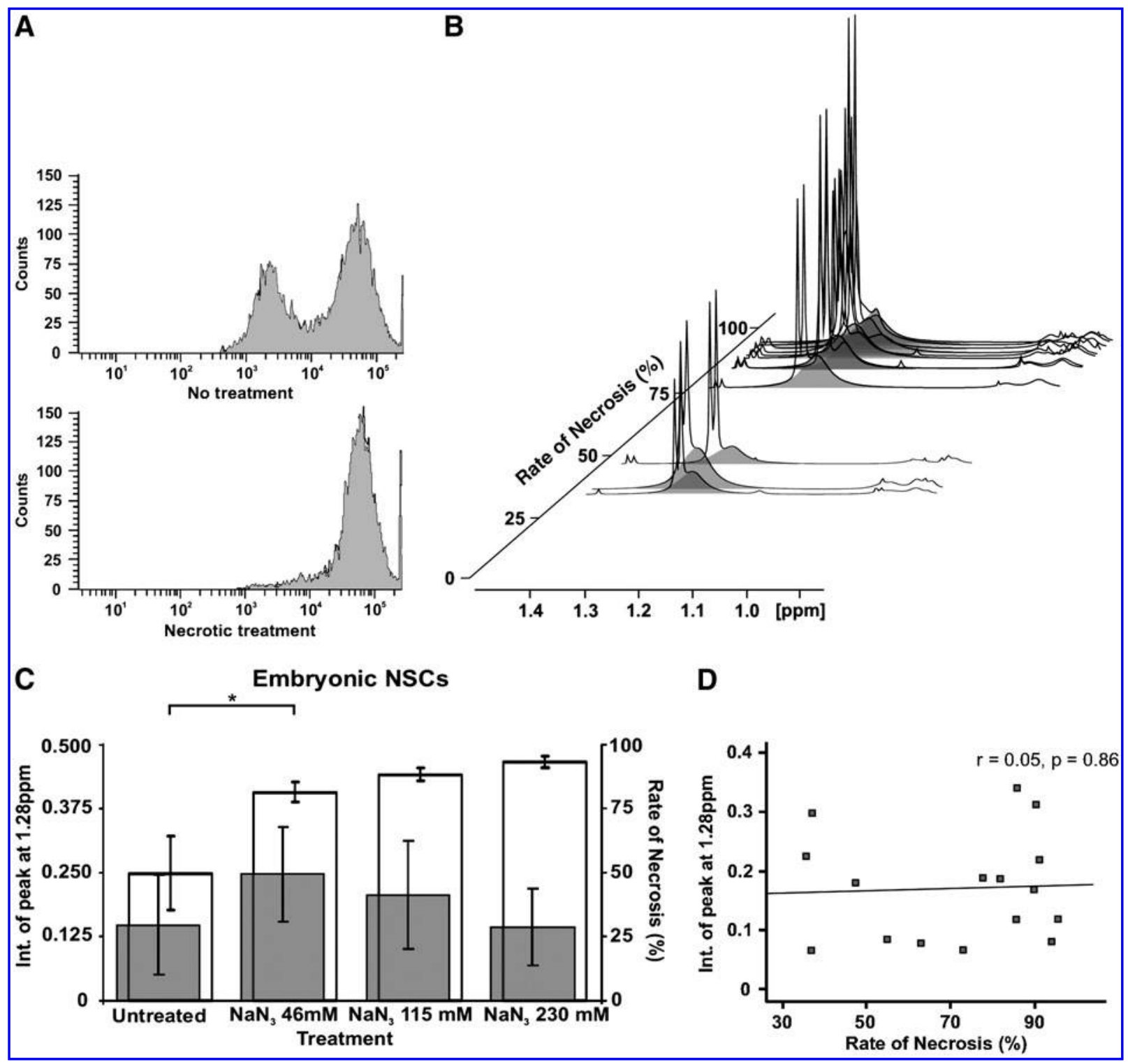

FIG. 6. Effects of necrotic cell death on integral intensities at $1.28 \mathrm{ppm}$ in proton magnetic resonance spectroscopy $\left({ }^{1} \mathrm{H}-\right.$ MRS). (A) Representative examples of an untreated and a treated culture of embryonic neural stem cells (NSCs). Necrosis was induced using sodium azide. The increase in cell fluorescence due to the permeabilization of the necrotic cell membrane was measured by fluorescence-activated cell sorting (FACS) analysis. (B) ${ }^{1} \mathrm{H}-\mathrm{MRS}$ spectra of various cultures of embryonic NSCs are displayed as a function of their necrotic rate, either spontaneous, or due to exposure to sodium azide. (C) Integral intensities at $1.28 \mathrm{ppm}$ of embryonic NSCs with different levels of necrosis are displayed by the grey bars, referenced to the $y$ axis on the left side of the graph. Necrosis was induced by increasing concentrations of sodium azide, as indicated. The resulting level of necrosis is displayed by the recessed white bars with reference to the $y$ axis on the right side of the graph. $\left({ }^{*}\right)$ Significant differences with $p<0.05$. (D) Correlation of the level of necrosis in embryonic NSCs with integral intensities at $1.28 \mathrm{ppm}$. In contrast to apoptosis, no significant correlation between the two parameters could be found (Pearson correlation coefficient). $\mathrm{NaN}_{3}$, Sodium azide.

leading to the differences in apoptosis rates and sensitivity to apoptotic stressors remain unknown, but are probably at least in part cell-intrinsic processes. Additionally, the high variance in apoptosis rates might also be induced by the cell preparation needed for MRS measurements, whereas MRS itself does not induce apoptosis (see Materials and Methods for details). Our study extended markedly the previous knowledge by measuring peak intensities of a very wide range of cells at very different apoptotic levels (quantified by PI-FACS) and analysis by a linear multiple regression model, which clearly demonstrated that the 1.28-ppm signal is specifically correlated with the level of apoptosis, but not with necrosis, cell size, or cell type.

The discrepancies between the two recent studies and the in vitro studies reported by Manganas and co-workers describing the 1.28-ppm peak as highly enriched and thus specific for NSCs and other neuroectodermal progenitor populations ${ }^{10}$ could be related to different rates of apoptosis in the cultures used. We prepared our NSC cultures under close adherence to the protocol provided by Manganas et al. ${ }^{10}$ 
and measured considerable levels of at least $9 \%$ of spontaneous apoptosis in these cultures, accompanied by a corresponding signal at $1.28 \mathrm{ppm}$. Unfortunately, Manganas and co-workers did not measure apoptosis or cell death in their study. Consistently, cell types that were used as negative controls by Manganas and colleagues, such as astrocytes or differentiated neural cell cultures, have a known resistance to spontaneous and induced apoptosis and also displayed a low intensity or absence of the 1.28-ppm peak in our study. ${ }^{32}$ The specificity for apoptosis of the peak at $1.28 \mathrm{ppm}$ is further supported by the finding that even NSC cultures with very low levels of cell death do not display the peak at $1.28 \mathrm{ppm}^{14}$

A recent publication focusing on methodological aspects of spectrum analysis did not completely rule out that in the spectral region of interest around $1.28 \mathrm{ppm}$ there are both a NSC-specific peak at low signal intensity and already known peaks derived from mobile methylene groups. ${ }^{33}$ However, evidence for the existence of two distinct signals in the same spectral region was neither found by the cited experimental study on cell-specificity of the signal nor by our results. ${ }^{14}$

Apoptosis is a proliferation-related event, because cells have to be in the active phases of the cell cycle to undergo programmed cell death. ${ }^{34-36}$ Additional analysis revealed no correlation between the intensity of the peak at $1.28 \mathrm{ppm}$ and other phases of the cell cycle, whereas for the $\mathrm{CH} 2 / \mathrm{CH} 3$ ratio a mild negative correlation could be found with the proportion of the cells in $G_{0} / G_{1}$ phase. However, this correlation was much weaker than the correlation between $\mathrm{CH} 2 / \mathrm{CH} 3$ and apoptosis $(r=-0.401$ versus $r=0.784$, Pearson correlation). These data further support that the peak at $1.28 \mathrm{ppm}$ is related primarily to apoptosis, because apoptotic cells have been found in this and other studies to recruit mainly from the cell population in $G_{0} / G_{1}$ phase, so that the mild negative correlation can be viewed as an indirect effect. $^{37}$

Cultures of quickly proliferating cell populations, such as tumor or stem cells, are particularly prone to induction of apoptosis, which can be favored even by scarcity of nutrition or contact inhibition characteristic for quickly proliferating cultures. Thus, there is no need for a second, cell-specific signal in this spectral region to explain the differences in peak intensity observed in different cultures. In our study, NSCs were the most sensitive to spontaneous and toxic induction of apoptosis, and this suffices to explain the high intensity of the peak in NSC cultures. The former study by Ramm and colleagues and our study cannot fully rule out the existence of a NSC-specific signal in the same spectral region subjacent to the apoptosis-related 1.28-ppm peak. ${ }^{14}$ This seems however very unlikely, because the peak was not detectable in NSC cultures with very low levels of cell death. ${ }^{14}$

Our results are well in line with a considerable number of experimental and clinical studies on the origin and biological significance of the spectroscopic signal from lipid-derived methylene groups. The signal has been linked to an increased mobility of methylene groups in membrane lipids as the result of the conformation of so called internal lipid bodies, a process strongly related to apoptotic cell death, but also to other rather cell type-independent biological processes such as inflammation, malignant proliferation, or necrosis. ${ }^{16}$ The link of the signal has been best established for apoptosis and has found entry into clinical diagnostic procedures, such as the evaluation of tumor regression or type. ${ }^{38-40}$ The two tumor cell types studied in the present report, mouse melanoma and glioma cells, were also prone to the induction of apoptosis and depicted a concomitant high peak intensity.

Manganas and co-workers additionally used two models to claim the usefulness of the 1.28-ppm signal for studying NSC behavior in vivo. First, NSCs were transplanted into rat brains and subsequently detected by the peak at $1.28 \mathrm{ppm}$. However, this paradigm seems to be unsuitable for distinguishing between the in vivo detection of a cell-specific signal and the detection of an apoptosis-derived signal in the same spectral region, because it has been shown that the majority of transplanted cells do not survive the procedure of transplantation (up to $96 \%$ ) and undergo apoptosis or necrosis. ${ }^{41}$ The second model used was electroconvulsive shock treatment as a potent inductor of neurogenesis. This paradigm is not only a potent inductor of NSCs proliferation and their nonfunctional differentiation, but also of apoptotic cell death of the majority of newborn cells, ${ }^{42,43}$ thus it is not able to provide a clear distinction between a NSC-specific and an apoptosis-specific MRS signal. It is noted that these limitations do not necessarily disable the method for studying neurogenesis in vivo, because estimating the rate of apoptosis within the neurogenic niche might be an indirect measure of stem cell turnover or homeostasis as supported by the results of Manganas and co-workers. ${ }^{10}$

Together, our study provides evidence that the peak at $1.28 \mathrm{ppm}$ in ${ }^{1} \mathrm{H}-\mathrm{MRS}$ spectra is a specific marker for apoptosis, and its intensity neither correlates with a distinct cell type, such as NSCs, nor with other cellular states, such as necrosis or cell cycle phase distribution. Because the apoptotic cell death is a biological process closely related to stem cell functioning and homeostasis, our results do not exclude a relevance of this signal in studying NSC biology in vivo as reported by Manganas and colleagues. ${ }^{10}$ Because in vivo ${ }^{1} \mathrm{H}$ MRS provides spatially resolved spectral information, it could be used to obtain indirect information about the regulation and homeostasis of the NSC pool within neurogenic niches by studying the local apoptosis rate.

\section{Acknowledgments}

The authors express their grateful thanks to Eberhard Kuhlisch, Dresden University of Technology, for help in statistics, Claudia Waskow, Center for Regenerative Therapies Dresden, for methodological cell culture advice, and Oliver Speck, Otto von Guericke-Universität Magdeburg, for help with high-field ${ }^{1} \mathrm{H}$-MRS. Induced pluripotent stem cells were kindly provided by Hans R. Schöler, Max Planck Institute for Molecular Biomedicine, Department of Cell and Developmental Biology, Münster, Germany. The authors would like to thank Susann Höfner, Sylvia Kanzler, Andrea Kempe, and Cornelia May for excellent technical assistance. The work was supported in part by the Medical Faculty Research Program MeDDrive to A.H. and M.B., and by the Deutsche Forschungsgemeinschaft (DFG) through the Collaborative Research Center 655: Cells into tissues: stem cell and progenitor commitment and interactions during tissue formation" (SFB 655 Dresden, project A23) to A.S., and the DFG-Research Center for Regenerative Therapies Dresden (CRTD) to K.L., A.H., M.B., and A.S. 


\section{Author Contributions}

Kai F. Lowenbrück: Conception and design, collection and/or assembly of data, co-principal investigator and grant support, data analysis and interpretation, writing and revision of manuscript. Beate Fuchs: Design, collection and/or assembly of data, data analysis and interpretation, manuscript writing. Andreas Hermann: Conception and design, provision of study material, collection and/or assembly of data, data analysis and interpretation, manuscript writing. Moritz Brandt: Collection and/or assembly of data, data analysis and interpretation, revising of manuscript. Annett Werner: Collection and/or assembly of data, data analysis and interpretation, revising of manuscript. Sigrid Schwarz: Collection and/or assembly of data, revising of manuscript. Matthias Kirsch: Provision of study material. Johannes Schwarz: Collection and/or assembly of data, revising of manuscript. Jürgen Schiller: Conception and design, coprincipal investigator, data analysis and interpretation, manuscript writing. Alexander Storch: Conception and design, co-principal investigator and grant support, data analysis and interpretation, writing, and revision of manuscript.

\section{Author Disclosure Statement}

No competing financial interests exist.

\section{References}

1. Gage FH, Ray J, Fisher LJ. Isolation, characterization, and use of stem cells from the CNS. Annu Rev Neurosci 1995;18: 159-192.

2. Kempermann G, Kuhn HG, Gage FH. More hippocampal neurons in adult mice living in an enriched environment. Nature 1997;386:493-495.

3. van Praag H, Kempermann G, Gage FH. Running increases cell proliferation and neurogenesis in the adult mouse dentate gyrus. Nat Neurosci 1999;2:266-270.

4. Alvarez-Buylla A. Neurogenesis and plasticity in the CNS of adult birds. Exp Neurol 1992;115:110-114.

5. Storch A, Schwarz J. Neural stem cells and neurodegeneration. Curr Opin Investig Drugs 2002;3:774-781.

6. Emsley JG, Mitchell BD, Kempermann G, Macklis JD. Adult neurogenesis and repair of the adult CNS with neural progenitors, precursors, and stem cells. Prog Neurobiol 2005;75: 321-341.

7. Greenberg DA, Jin K. Neurodegeneration and neurogenesis: Focus on Alzheimer's disease. Curr Alzheimer Res 2006;3: 25-28.

8. Curtis MA, Kam M, Nannmark U, Anderson MF, Axell MZ, Wikkelso C, Holtas S, van Roon-Mom WMC, Bjork-Eriksson T, Nordborg C, Frisen J, Dragunow M, Faull RLM, Eriksson PS. Human neuroblasts migrate to the olfactory bulb via a lateral ventricular extension. Science 2007;315:1243-1249.

9. Eriksson PS, Perfilieva E, Bjork-Eriksson T, Albron A-M, Nordborg C, Peterson DA, Gage FH. Neurogenesis in the adult human hippocampus. Nat Med 1998;4:1313-1317.

10. Manganas LN, Zhang X, Li $Y$, Hazel RD, Smith SD, Wagshul ME, Henn F, Benveniste H, Djuric PM, Enikolopov G, Maletic-Savatic M. Magnetic resonance spectroscopy identifies neural progenitor cells in the live human brain. Science 2007;318:980-985.

11. Friedman SD. Comment on "Magnetic resonance spectroscopy identifies neural progenitor cells in the live human brain." Science 2008;321:640; author reply 640.
12. Hoch JC, Maciejewski MW, Gryk MR. Comment on "magnetic resonance spectroscopy identifies neural progenitor cells in the live human brain." Science 2008;321:640; author reply 640 .

13. Jansen JF, Gearhart JD, Bulte JW. Comment on "Magnetic resonance spectroscopy identifies neural progenitor cells in the live human brain." Science 2008;321:640; author reply 640.

14. Ramm P, Couillard-Despres S, Plotz S, Rivera FJ, Krampert M, Lehner B, Kremer W, Bogdahn U, Kalbitzer HR, Aigner L. A nuclear magnetic resonance biomarker for neural progenitor cells: Is it all neurogenesis? Stem Cells 2009;27: 420-423.

15. Callies R, Sri-Pathmanathan RM, Ferguson DY, Brindle KM. The appearance of neutral lipid signals in the $1 \mathrm{H}$ NMR spectra of a myeloma cell line correlates with the induced formation of cytoplasmic lipid droplets. Magn Reson Med 1993;29:546-550.

16. Hakumaki JM, Kauppinen RA. 1H NMR visible lipids in the life and death of cells. Trends Biochem Sci 2000;25:357-362.

17. Remy C, Fouilhe N, Barba I, Sam-Lai E, Lahrech H, Cucurella M-G, Izquierdo M, Moreno A, Ziegler A, Massarelli R, Decorps M, Arus C. Evidence that mobile lipids detected in rat brain glioma by $1 \mathrm{H}$ nuclear magnetic resonance correspond to lipid droplets. Cancer Res 1997;57:407-414.

18. Blankenberg FG, Katsikis PD, Storrs RW, Beaulieu C, Spielman D, Chen JY, Naumovski L, Tait JF. Quantitative analysis of apoptotic cell death using proton nuclear magnetic resonance spectroscopy. Blood 1997;89:3778-3786.

19. Milosevic J, Maisel M, Wegner F, Leuchtenberger J, Wenger RH, Gerlach M, Storch A, Schwarz J. Lack of hypoxiainducible factor-1 alpha impairs midbrain neural precursor cells involving vascular endothelial growth factor signaling. J Neurosci 2007;27:412-421.

20. Freudenberg U, Hermann A, Welzel PB, Stiri K, Schwarz SC, Grimmer M, Zieris A, Panyanuwat W, Zschoche S, Meinhold D, Storch A, Werner C. A star-PEG-heparin hydrogel platform to aid cell replacement therapies for neurodegenerative diseases. Biomaterials 2009;30:5049-5060.

21. Song H, Stevens CF, Gage FH. Astroglia induce neurogenesis from adult neural stem cells. Nature 2002;417:39-44.

22. Kawasaki H, Mizuseki K, Nishikawa S, Kaneko, S, Kuwana Y, Nakanishi S, Nishikawa S-I, Sasai Y. Induction of midbrain dopaminergic neurons from ES cells by stromal cellderived inducing activity. Neuron 2000;28:31-40.

23. Kim JB, Sebastiano V, Wu G, Arauzo-Bravo MJ, Sasse P, Gentile L, Ko K, Ruau D, Ehrich M, van den Boom D, Meyer J, Hubner K, Bernemann C, Ortmeier C, Zenke M, Fleischmann BK, Zaehres H, Scholer HR. Oct4-induced pluripotency in adult neural stem cells. Cell 2009;136:411-419.

24. Heller A, Price S, Won L. Glial-derived neurotrophic factor (GDNF) induced morphological differentiation of an immortalized monoclonal hybrid dopaminergic cell line of mesencephalic neuronal origin. Brain Res 1996;725:132-136.

25. Kirsch M, Weigel P, Pinzer T, Carroll RS, Black PM, Schackert H-K, Schackert G. Therapy of hematogenous melanoma brain metastases with endostatin. Clin Cancer Res2005;11:1259-1267.

26. Kirsch M, Allende R, Black P, Schackert G. Endogenous growth inhibition of angiogenesis in brain tumors. Cancer Metastasis Rev 2007;26:469-479.

27. Uo T, Kinoshita Y, Morrison RS. Apoptotic actions of p53 require transcriptional activation of PUMA and do not involve a direct mitochondrial/cytoplasmic site of action in postnatal cortical neurons. J Neurosci 2007;27:12198-12210. 
28. Lizard G, Fournel S, Genestier L, Dhedin N, Chaput C, Flacher M, Mutin M, Panaye G, Revillard J-P. Kinetics of plasma membrane and mitochondrial alterations in cells undergoing apoptosis. Cytometry 1995;21:275-283.

29. Nicoletti I, Migliorati G, Pagliacci MC, Grignani F, Riccardi C. A rapid and simple method for measuring thymocyte apoptosis by propidium iodide staining and flow cytometry. J Immunol Methods 1991;139:271-279.

30. Glick B, LaVia MF, Koger B. Cell flow cytometry of fixed and unfixed bursal and thymic cells. Poult Sci 1985;64:737-743.

31. Urenjak J, Williams SR, Gadian DG, Noble M. Proton nuclear magnetic resonance spectroscopy unambiguously identifies different neural cell types. J Neurosci 1993;13:981-989.

32. Morris EJ, Geller HM. Induction of neuronal apoptosis by camptothecin, an inhibitor of DNA topoisomerase-I: Evidence for cell cycle-independent toxicity. J Cell Biol 1996; 134:757-770.

33. Dong Z, Dreher W, Leibfritz D, Peterson BS. Challenges of using MR spectroscopy to detect neural progenitor cells in vivo. AJNR Am J Neuroradiol 2009;30:1096-1101.

34. Hallstrom TC, Nevins JR. Balancing the decision of cell proliferation and cell fate. Cell Cycle 2009;8:532-535.

35. Pawlik TM, Keyomarsi K. Role of cell cycle in mediating sensitivity to radiotherapy. Int J Radiat Oncol Biol Phys 2004;59:928-942.

36. Schmitt E, Paquet C, Beauchemin M, Bertrand R. DNAdamage response network at the crossroads of cell-cycle checkpoints, cellular senescence and apoptosis. J Zhejiang Univ Sci B 2007;8:377-397.

37. Zhang Q, Chen Y, Wang B-D, He P, Su YA. Differences in apoptosis and cell cycle distribution between human melanoma cell lines UACC903 and UACC903(+6), before and after UV irradiation. Int J Biol Sci 2007;3:342-348.

38. Bathen TF, Jensen LR, Sitter B, Fjosne HE, Halgunset J, Axelson DE, Gribbestad IS, Lundgren S. MR-determined metabolic phenotype of breast cancer in prediction of lymphatic spread, grade, and hormone status. Breast Cancer Res Treat 2007;104:181-189.
39. Hakumaki JM, Poptani H, Sandmair AM, Yla-Herttuala S, Kauppinen RA. 1H MRS detects polyunsaturated fatty acid accumulation during gene therapy of glioma: implications for the in vivo detection of apoptosis. Nat Med 1999;5:13231327.

40. Sjobakk TE, Johansen R, Bathen TF, Sonnewald U, Kvistad K, Lundgren S, Gribbestad I. Metabolic profiling of human brain metastases using in vivo proton MR spectroscopy at 3T. BMC Cancer 2007;7:141.

41. Muraoka K, Shingo T, Yasuhara T, Kameda M, Yuan W, Hayase H, Matsui T, Miyoshi Y, Date I. The high integration and differentiation potential of autologous neural stem cell transplantation compared with allogeneic transplantation in adult rat hippocampus. Exp Neurol 2006;199:311-327.

42. Ekdahl CT, Zhu C, Bonde S, Bahr BA, Blomgren K, Lindvall O. Death mechanisms in status epilepticus-generated neurons and effects of additional seizures on their survival. Neurobiol Dis 2003;14:513-523.

43. Ekdahl CT, Mohapel P, Weber E, Bahr B, Blomgren K, Lindvall O. Caspase-mediated death of newly formed neurons in the adult rat dentate gyrus following status epilepticus. Eur J Neurosci 2002;16:1463-1471.

Address correspondence to: Alexander Storch

Dresden University of Technology

Department of Neurology and Center for Regenerative

Therapies Dresden (CRTD)

Fetscherstrasse 74

01307 Dresden

Germany

E-mail: alexander.storch@neuro.med.tu-dresden.de

Received: August 15, 2010

Accepted February 15, 2011 